\title{
MODELING STRONG MOTIONS PRODUCED BY EARTHQUAKES WITH TWO-DIMENSIONAL NUMERICAL CODES
}

\author{
By Donald V. Helmberger and John E. Vidale
}

\begin{abstract}
We present a scheme for generating synthetic point-source seismograms for shear dislocation sources using line source (two-dimensional) theory. It is based on expanding the complete three-dimensional solution of the wave equation expressed in cylindrical coordinates in an asymptotic form which provides for the separation of the motions into SH and P-SV systems. We evaluate the equations of motion with the aid of the Cagniard-de Hoop technique and derive close-formed expressions appropriate for finite-difference source excitation.
\end{abstract}

\section{INTRODUCTION}

Recent strong motion modeling efforts have been restricted to plane-layered models as displayed in Figure 1a. Point-source shear dislocations, or double couples, are applied at each element where the seismic field is decomposed into $S H$ and $P$ $S V$ type motions and the vertical and horizontal dependences separated following the approach pioneered by Harkrider (1964). Nonuniform fault slip may be simulated by summing weighted point sources distributed along the fault plane to construct realistic synthetic seismograms. Recent inversion studies based on matching these synthetics to observations such as Hartzell and Heaton (1983), Archuleta (1984), and Olson and Apsel (1982) have provided amazing detail on the complex faulting process for the Imperial Valley 1979 earthquake. Unfortunately, most geologic structures in the vicinity of earthquakes are at least as complicated as displayed in Figure 1b. Separating propagational effects from complex faulting becomes much more difficult in these situations.

In this paper, we address the construction of synthetics along the surface for twodimensional structures such as displayed in Figure 1b. We assume that the model remains constant into and out of the plane of the paper along with line sources through each element. We design the line-source characteristics to mimic the vertical radiation pattern appropriate for double couples, where the $S H$ and $P-S V$ field remain decoupled along paths to the receivers. Our main objective is to derive these line-source excitation functions.

In a companion paper [Vidale and Helmberger (1988)], we discuss numerical strong ground motion calculations for a two-dimensional structural model through the Los Angeles region and compare these results with observations from the San Fernando earthquake.

\section{THEORY}

The approach follows closely the usual shear dislocation theory developed for treating plane layered models, where the wave field is separated into vertical and horizontal functions. This separation is essential for expressing the field in terms of $S H$ and $P-S V$ systems and provides the key to our approach. A particularly convenient form of the solution is given by Helmberger and Harkrider (1977) in terms of Laplace-transformed displacements along the vertical, tangential, and radial directions, 


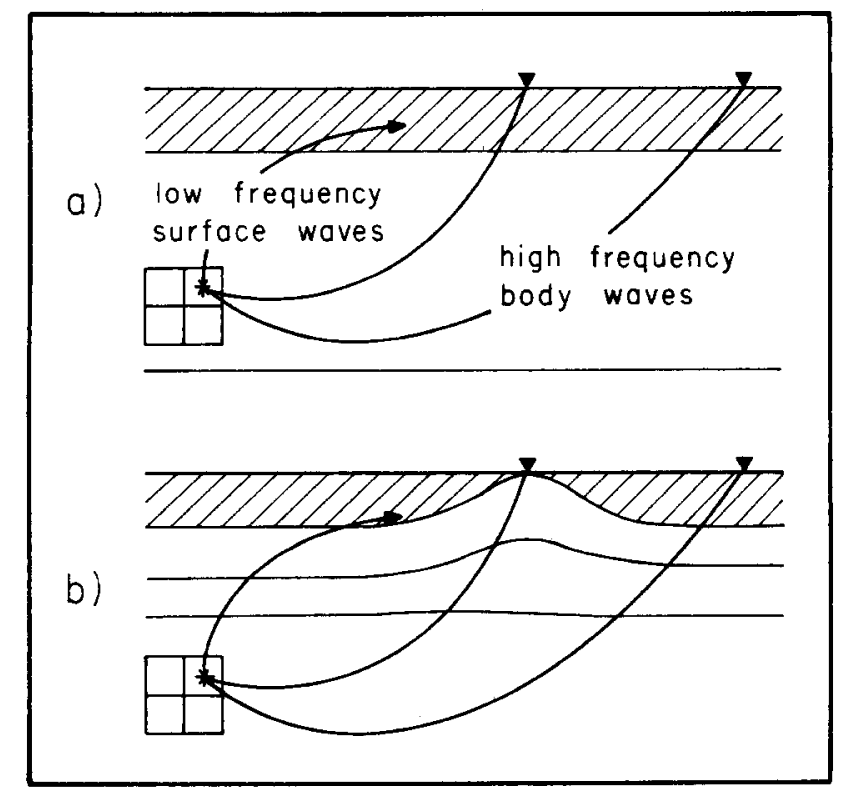

FIG. 1. Schematic diagram displaying energy paths for (a) flat-layered model versus (b) laterally varying structure. The model is two-dimensional or constant properties into and out of the plane of the paper.

$$
\begin{aligned}
& \hat{\boldsymbol{W}}=\frac{\partial \hat{\phi}}{\partial z}+s p \hat{\Omega} \\
& \hat{\boldsymbol{V}}=\frac{1}{r} \frac{\partial \hat{\phi}}{\partial \theta}-\frac{1}{s p r} \frac{\partial^{2} \hat{\Omega}}{\partial z \partial \theta}-\frac{\partial \hat{\chi}}{\partial r} \\
& \hat{\boldsymbol{Q}}=\frac{\partial \hat{\phi}}{\partial r}-\frac{1}{s p} \frac{\partial^{2} \hat{\Omega}}{\partial r \partial z}+\frac{1}{r} \frac{\partial \hat{\chi}}{\partial \theta}
\end{aligned}
$$

where $z, r$, and $\theta$ are the vertical, radial, polar angle coordinates, respectively. The $P$ wave potential $(\phi)$, the $S V$ wave potential $(\Omega)$, and the $S H$ wave potential $(\chi)$ for a strike-slip orientation are

$$
\begin{gathered}
\hat{\phi}=\frac{M_{0}}{4 \pi \rho} \frac{2}{\pi} \operatorname{Im} \int_{c}^{i \infty+c}\left(-p^{2}\right) \frac{p}{\eta_{\alpha}} \exp \left(-s \eta_{\alpha}|z-h|\right) K_{2}(s p r) d p \cdot \sin 2 \theta \\
\hat{\Omega}=\frac{M_{0}}{4 \pi \rho} \frac{2}{\pi} \operatorname{Im} \int_{c}^{i \infty+c}\left(-\epsilon p \eta_{\beta}\right) \frac{p}{\eta_{\beta}} \exp \left(-s \eta_{\beta}|z-h|\right) K_{2}(s p r) d p \cdot \sin 2 \theta \\
\hat{\chi}=\frac{M_{0}}{4 \pi \rho} \frac{2}{\pi} \operatorname{Im} \int_{c}^{i \infty+c}\left(\frac{1}{\beta^{2}}\right) \frac{p}{\eta_{\beta}} \exp \left(-s \eta_{\beta}|z-h|\right) K_{2}(s p r) d p \cdot \cos 2 \theta
\end{gathered}
$$


where

$s=$ Laplace transform variable

$p=$ ray parameter

$\eta_{v}=\left(1 / v^{2}-p^{2}\right)^{1 / 2}$

$h=$ depth of source

$\alpha=$ compressional velocity

$\beta=$ shear velocity

$\rho=$ density

$M_{0}=$ seismic moment

$c=$ a small constant that offsets path of integration from the imaginary axis, and

where

$$
\begin{aligned}
& \epsilon=\left\{\begin{array}{ll}
+1 & z>h \\
-1 & z<h
\end{array}\right. \text {, and } \\
& \theta=\text { strike from the end of the fault plane. }
\end{aligned}
$$

The geometry displaying the orientation of the fault in the cylindrical coordinate system is given in Figure 2. Note that a strike-slip event is defined by $\lambda=0^{\circ}$ and $\delta=90^{\circ}$. Integrals of the type given in equations (2) through (4) can be transformed back into the time domain by applying the Cagniard-de Hoop technique as discussed by Helmberger and Harkrider (1977). An asymptotic solution and one useful for our purpose can be obtained by expanding the modified Bessel functions

$$
K_{2}(s p r)=\sqrt{\frac{\pi}{2} \operatorname{spr}} e^{-s p r}\left[1+\frac{15}{8 s p r}+\cdots\right]
$$

substituting the resulting power series in potentials back into the displacements, expression (1), and expand again in powers of $(1 / s)$. The first term of such an

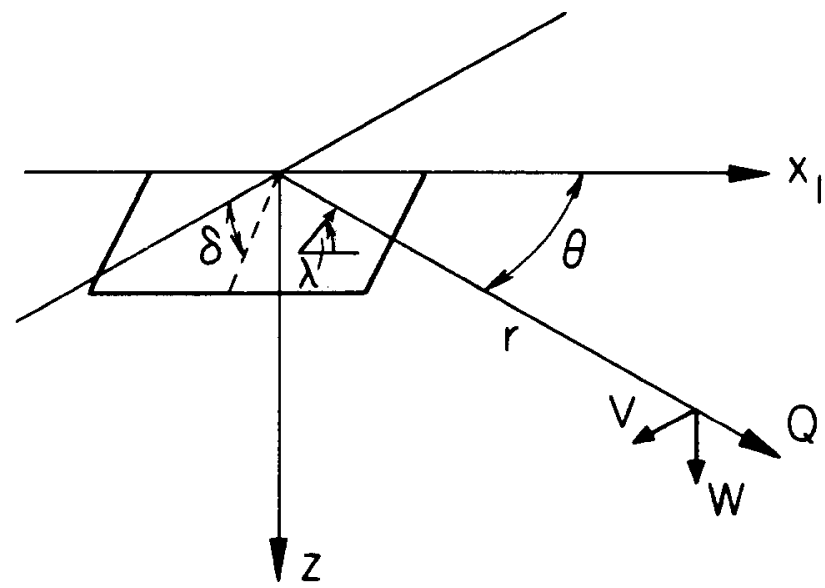

FIG. 2. Description of conventions for mechanism and orientation. 
expression has the following form

$$
\begin{aligned}
& \boldsymbol{W}=\frac{M_{0}}{4 \pi \rho} \frac{d}{d t}\left[\dot{D}(t) *\left(w_{\alpha}+w_{\beta}\right)\right] \sin 2 \theta \\
& \boldsymbol{V}=\frac{M_{0}}{4 \pi \rho} \frac{d}{d t}\left[\dot{D}(t) * v_{\beta}\right] \cos 2 \theta \\
& \boldsymbol{Q}=\frac{M_{0}}{4 \pi \rho} \frac{d}{d t}\left[\dot{D}(t) *\left(q_{\alpha}+q_{\beta}\right)\right] \sin 2 \theta
\end{aligned}
$$

where

$$
\begin{aligned}
& w_{\alpha}=\sqrt{\frac{2}{r}} \frac{1}{\pi}\left[\frac{1}{\sqrt{t}} * \operatorname{Im}\left(\frac{\sqrt{p}}{\eta_{\alpha}}\left(-p^{2}\right)\left(-\epsilon \eta_{\alpha}\right) \frac{d p}{d t}\right)\right] \\
& w_{\beta}=\sqrt{\frac{2}{r}} \frac{1}{\pi}\left[\frac{1}{\sqrt{t}} * \operatorname{Im}\left(\frac{\sqrt{p}}{\eta_{\beta}}\left(-\epsilon p \eta_{\beta}\right)(p) \frac{d p}{d t}\right)\right] \\
& v_{\beta}=\sqrt{\frac{2}{r}} \frac{1}{\pi}\left[\frac{1}{\sqrt{t}} * \operatorname{Im}\left(\frac{\sqrt{p}}{\eta_{\beta}}\left(\frac{1}{\beta^{2}}\right)(p) \frac{d p}{d t}\right)\right] \\
& q_{\alpha}=\sqrt{\frac{2}{r}} \frac{1}{\pi}\left[\frac{1}{\sqrt{t}} * \operatorname{Im}\left(\frac{\sqrt{p}}{\eta_{\alpha}}\left(-p^{2}\right) p \frac{d p}{d t}\right)\right] \\
& q_{\beta}=\sqrt{\frac{2}{r}} \frac{1}{\pi}\left[\frac{1}{\sqrt{t}} * \operatorname{Im}\left(\frac{\sqrt{p}}{\eta_{\beta}}\left(-\epsilon p \eta_{\beta}\right)\left(-\epsilon \eta_{\beta}\right) \frac{d p}{d t}\right)\right] .
\end{aligned}
$$

This is a first term asymptotic expansion similar to the expansion used for explosive sources, see Helmberger (1968). The approximation is accurate for $s p r \gg 1$, which means it is most accurate for high-frequency, large range, and nonvertical take-off angles. The two arrivals in the $w_{j}$ and $q_{j}$ cases are the $P$ wave and $S V$ wave. Note that the first term becomes uncouples in the $V$ depends only on $\chi$, and $W$ and $Q$ only on $\phi$ and $\Omega$; so, the $S H$ solution separates from the $P-S V$ solution in this asymptotic form.

Suppose we now consider the field variable given by

$$
\Psi=\sqrt{\frac{2}{r}} \frac{1}{\pi}\left[\frac{1}{\sqrt{t}} * \operatorname{Im}\left(\frac{\sqrt{p}}{\eta_{v}} \frac{d p}{d t}\right)\right]
$$

which has a simple interpretation following the Cagniard-de Hoop technique, where

$$
\begin{aligned}
& p=\frac{r}{R^{2}} t+i\left(t^{2}-\frac{R^{2}}{V^{2}}\right)^{1 / 2} \frac{|z|}{R^{2}} \\
& \eta_{v}=\frac{|z|}{R^{2}} t-i\left(t^{2}-\frac{R^{2}}{V^{2}}\right)^{1 / 2} \frac{r}{R^{2}}
\end{aligned}
$$


and

$$
\frac{d p}{d t}=i \eta_{V} H(t-R / V) /\left(t^{2}-\frac{R^{2}}{V^{2}}\right)^{1 / 2},
$$

where $V$ can be either $\alpha$ or $\beta$. Substituting these equations into (14) yields

$$
\Psi \approx \frac{1}{R} H(t-R / V)
$$

where $R=\left(r^{2} p l z^{2}\right)^{1 / 2}$ and the imaginary parts of $p$ and $\eta_{V}$ have been neglected, namely

$$
\operatorname{Re}(p)=\sin \phi / V \text { and } \operatorname{Re}\left(\eta_{V}\right)=\cos \psi / V
$$

The $(p)$ becomes the well-known ray parameter and $\left(\eta_{V}\right)$ the vertical slowness. The extra $\left(p^{2}\right)$ occurring in $W_{\alpha}$ becomes the vertical radiation pattern and $\eta_{\alpha}$ the vertical component of the $P$ wave. The $(\epsilon)$ expresses the jump in polarity across the origin (see Figure 3a). This series of simplifications is called the first-motion approxima-

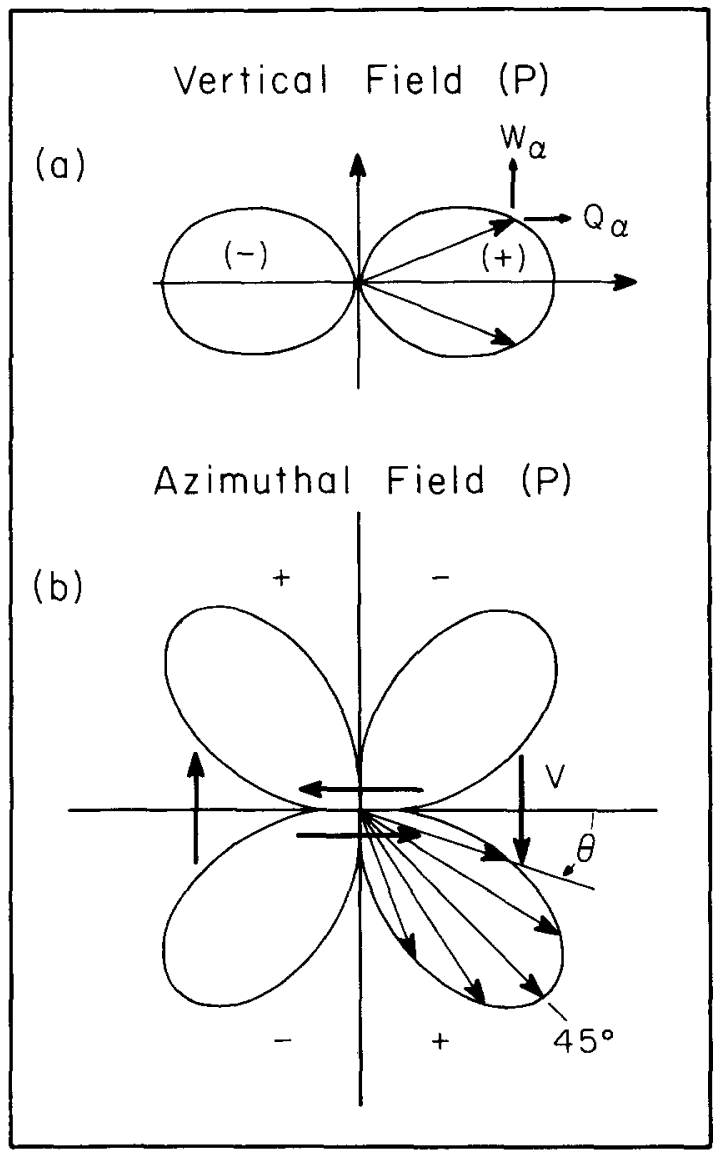

FIG. 3. The top panel displays the vertical radiation field appropriate for a strike-slip excited $P$ wave with a $\sin ^{2} \phi$ pattern. The bottom panel displays the azimuthal field showing the strong $P$ wave loading near $45^{\circ}$. Between the times $t_{\alpha}$ and $t_{\beta}$, the material in the fourth quadrant flows away from the maximum compression, namely clockwise for $\theta>45^{\circ}$ and counterclockwise for $\theta<45^{\circ}$. 
tions and has proven itself quite useful in modeling seismograms at teleseismic distances [see Langston and Helmberger (1975)].

A more accurate solution at local distances can be obtained by solving expressions (9) through (13) numerically and substituting into (6), (7), and (8), which yields the first term of the asymptotic displacement as discussed. The results for a wholespace model are displayed in Figure 4. Higher order terms, $(1 / s)^{n}$, are included along with the exact Cagniard-de Hoop solution on the bottom [see Helmberger and Harkrider (1977)]. Note the slight upturn for the 12-term solution at the end of the radial motion. The solution rises rapidly for longer times, and these asymptotics suffer the usual blow-up at large times characteristic of such series. Also, note that the so-called near-field terms are missing along the top row. The near-field contributions are particularly easy to see by noting that the first term of the tangential motion, $V$ of expression (1), depends on the azimuthal change of the $P$ wave. A schematic of the horizontal $P$ wave pattern is displayed in Figure 3b, indicating the compressional field peaked at $\theta=45^{\circ}$. Since the material at a receiver, as in Figure 4 , behaves elastically before the arrival of the shear wave, it relaxes by moving counterclockwise for angles less than $45^{\circ}$. Similar drifts occur for the other two components between the $P$ and $S$ wave arrivals, where each time the material moves to relax from the $P$ wave loading. This drifting should end upon arrival of the shear wave, which is well known from the exact solution. Unfortunately, the asymptotics continue to drift, which can cause severe problems at long periods, and should be avoided for source durations longer than a significant fraction of $\left(t_{\beta}-t_{\alpha}\right)$, the timing separation between the $P$ and $S$ waves.

Expressions (6) through (13) have produced the goal of separating the motions into vertical and horizontal functions, but we still must require that these expres-

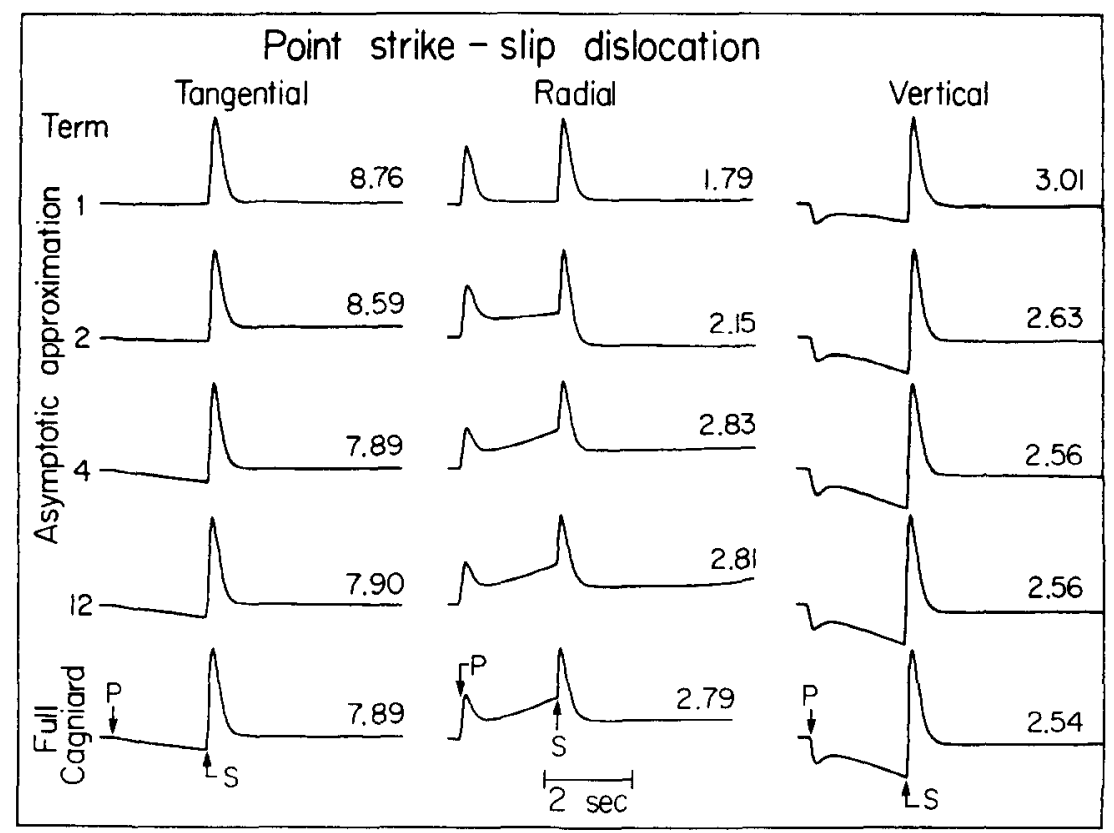

FIG. 4. Comparison of the three components of displacement for a whole-space with a strike-slip source. The top four rows contain the asymptotic summation with $1,2,4$, and 12 terms. The full solution is displayed at the bottom. The source depth is $8 \mathrm{~km}$, and the range is $16 \mathrm{~km}$. Model parameters are $\alpha=$ $6.2 \mathrm{~km} / \mathrm{sec}, \beta=3.5 \mathrm{~km} / \mathrm{sec}$, and $\rho=2.7 \mathrm{gm} / \mathrm{cm}^{3}$. 
sions be solutions of the two-dimensional wave equation. This requires removing the $\sqrt{p}$ dependence.

One approach is to expand $\sqrt{p}$ in a power series about some fixed direction, $p_{0}$,

$$
\sqrt{p}=\sqrt{p_{0}}+\frac{1}{2} \frac{1}{\sqrt{p_{0}}}\left(p-p_{0}\right)=\frac{\sqrt{p_{0}}}{2}+\frac{p}{2 \sqrt{p_{0}}}
$$

and carry the second factor as a correction term. The first term has shown to be adequate in, Vidale et al. (1985) where we approximated

$$
\sqrt{\frac{v p}{r}}=\frac{1}{\sqrt{R}}
$$

although we must avoid $p_{0}=0$ or $r=0$. The effective vertical radiation patterns then become the real-part operator, $\operatorname{Re}(\quad)$, of a product of complex $p$ 's and $\eta$ 's as defined in (15). For instance, from (9) we obtain

$$
\Psi^{1} \equiv \operatorname{Im}\left(\frac{1}{\eta_{\alpha}} \frac{d p}{d t}\left(-p^{2}\right)\left(-\epsilon \eta_{\alpha}\right)\right)=\operatorname{Re}\left(\epsilon p^{2} \eta_{\alpha}\right) H(t-R / \alpha)\left(t^{2}-\frac{R^{2}}{\alpha^{2}}\right)^{1 / 2}
$$

and after some complex algebra

$$
\operatorname{Re}\left(\epsilon p^{2} \eta_{\alpha}\right)=\frac{-\epsilon|z| t^{3}}{R^{6}}\left[\left[z^{2}-3 r^{2}+\frac{R^{2}}{t^{2} \alpha^{2}}\left(2 r^{2}-z^{2}\right)\right]\right] .
$$

Thus, the vertical displacement for an incoming $P$ wave from a strike-slip source becomes

$$
W_{\alpha}=\sqrt{\frac{2}{R \alpha}} \frac{1}{\pi}\left[\frac{1}{\sqrt{t}} * \Psi^{1}\right]
$$

where $\left(\Psi^{1}\right)$ solves the two-dimensional wave equation. Note that for times near the wave front, $t=R / \alpha$ and the function

$$
\operatorname{Re}\left(\epsilon p^{2} \eta_{\alpha}\right)=\frac{\cos \phi \sin ^{2} \phi \epsilon}{\alpha^{3}}
$$

which is the result obtained earlier from the first-motion approximation. To produce transparent source box conditions for the finite-difference requires a complete solution for all times and $\left(\Psi^{1}\right)$ serves this role. In short, the complex near-field terms which change their shape with time is required if we want to send out a simple $\sin ^{2} \theta$ pattern at larger distances. Similar expressions can be derived from equations (10) through (13) and explicit two-dimensional excitation functions determined by evaluating the $\operatorname{Re}(\quad)$ operators. The grid mechanics along with the other fundamental fault orientations are given in Vidale and Helmberger (1987). 
We will include these results here for completeness. Let

$$
\Gamma_{\gamma}=\sqrt{\frac{2}{\gamma}}\left(\frac{M_{0}}{4 \pi \rho_{0}}\right) \frac{1}{\pi}\left(M_{0} \times 10^{-20}\right) \text { and } T_{\gamma}=\frac{R^{2}}{t^{2} \gamma^{2}}
$$

where $\gamma=\alpha$ or $\beta$ expressed in kilometers/second, $R$ is in kilometers, and $M_{0}$ is in ergs. We will assume that $\dot{D}(t)=\delta(t)$ [slightly smoothed as discussed in Vidale $e t$ al. (1985)], or that the slip function across the fault corresponds to a Heavside step function. Also, we define superscripts 1, 2, and 3 to refer to strike-slip, dip-slip, and $45^{\circ}$ dip-slip fundamental fault orientations. With these definitions, the analytic source expressions used in defining the displacements in the grid become

(Strike-slip)

$$
\begin{aligned}
& \boldsymbol{W}_{\alpha}^{1}=-z\left(z^{2}-3 r^{2}+T_{\alpha}\left(2 r^{2}-z^{2}\right)\right) \Psi_{\alpha} \\
& \boldsymbol{W}_{\beta}^{1}=-z\left(3 r^{2}-z^{2}+T_{\beta}\left(z^{2}-2 r^{2}\right)\right) \Psi_{\beta} \\
& \boldsymbol{V}_{\beta}^{1}=\frac{r}{R^{2}} \frac{1}{t^{2} \beta^{2}} \Psi_{\beta} \\
& \boldsymbol{Q}_{\alpha}^{1}=r\left(r^{2}-3 z^{2}+3 T_{\alpha} z^{2}\right) \Psi_{\alpha} \\
& \boldsymbol{Q}_{\beta}^{1}=r\left(3 z^{2}-r^{2}+T_{\beta}\left(r^{2}-2 z^{2}\right)\right) \Psi_{\beta} .
\end{aligned}
$$

(Dip-slip)

$$
\begin{aligned}
& \boldsymbol{W}_{\alpha}^{2}=-r\left(2 r^{2}-6 z^{2}+T_{\alpha}\left(4 z^{2}-2 r^{2}\right)\right) \Psi_{\alpha} \\
& \boldsymbol{W}_{\beta}^{2}=-r\left(6 z^{2}-2 r^{2}+T_{\beta}\left(r^{2}-5 z^{2}\right)\right) \Psi_{\beta} \\
& \boldsymbol{V}_{\beta}^{2}=\frac{z}{R^{2}} \frac{1}{t^{2} \beta^{2}} \Psi_{\beta} \\
& \boldsymbol{Q}_{\alpha}^{2}=z\left(6 r^{2}-2 z^{2}+T_{\alpha}\left(2 z^{2}-4 r^{2}\right)\right) \Psi_{\alpha} \\
& \boldsymbol{Q}_{\beta}^{2}=z\left(2 z^{2}-6 r^{2}+T_{\beta}\left(5 r^{2}-z^{2}\right)\right) \Psi_{\beta} .
\end{aligned}
$$

$\left(45^{\circ}\right.$ dip-slip)

$$
\begin{aligned}
& \boldsymbol{W}_{\alpha}^{3}=-z\left(9 r^{2}-3 z^{2}+T_{\alpha}\left(z^{2}-8 r^{2}\right)\right) \Psi_{\alpha} \\
& \boldsymbol{W}_{\beta}^{3}=-z\left(3 z^{2}-9 r^{2}+T_{\beta}\left(6 r^{2}-3 z^{2}\right)\right) \Psi_{\beta} \\
& \boldsymbol{Q}_{\alpha}^{3}=r\left(9 z^{2}-3 r^{2}+T_{\alpha}\left(2 r^{2}-7 z^{2}\right)\right) \Psi_{\alpha} \\
& \boldsymbol{Q}_{\beta}^{3}=r\left(3 r^{2}-9 z^{2}+T_{\beta}\left(6 z^{2}-3 r^{2}\right)\right) \Psi_{\beta} .
\end{aligned}
$$

These expressions and functional form are compatible with Cartesian coordinates with the horizontal coordinate, $x$, replacing $r$ as discussed by Helmberger (1974). Thus, the aforementioned expressions can be used to define the desired line-source excitation [see Vidale and Helmberger (1987)] for the details of source loading. 


\section{P-SV VERTICAL RADIATION PATTERNS}

\section{Divergence}

Curl

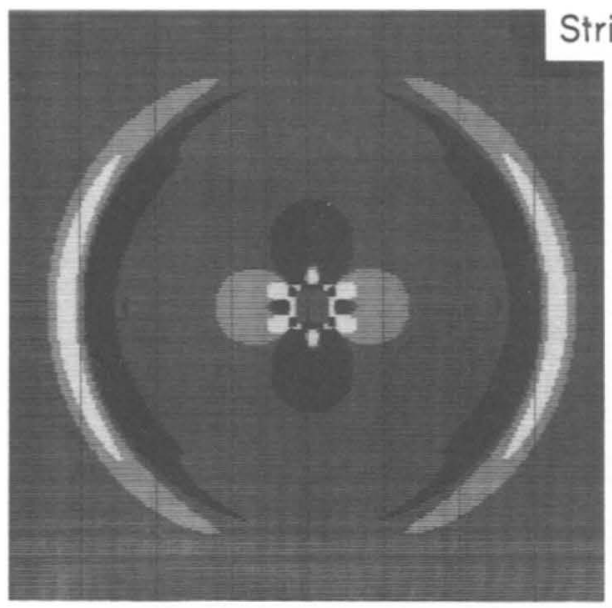

\section{Strike-Slip}

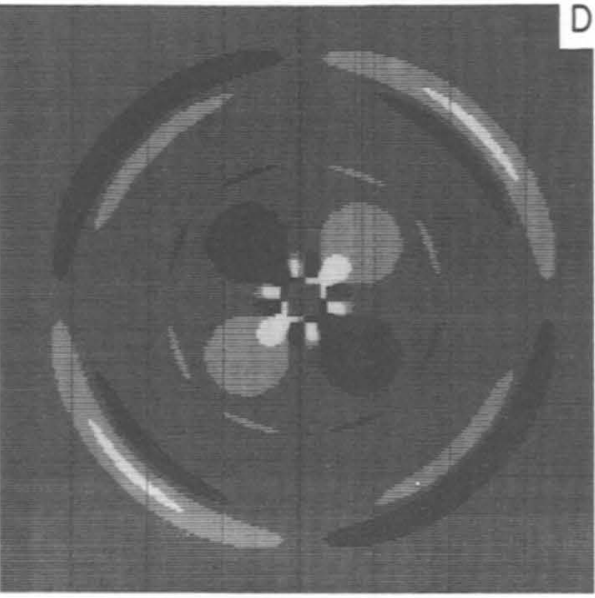

Dip-Slip
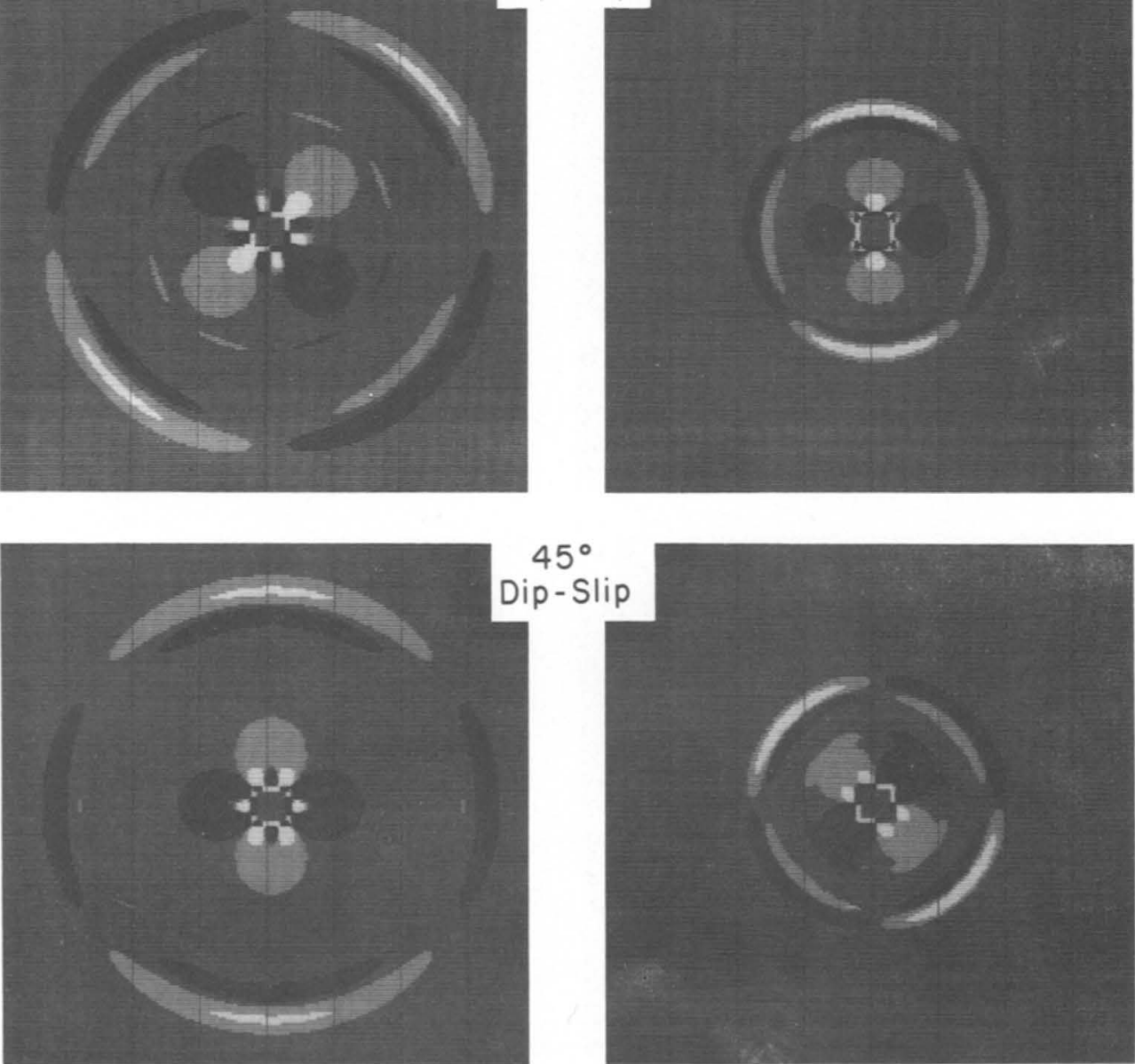

FIG. 5. $P-S V$ vertical radiation patterns. The divergence and curl fields due to strike-slip, dip-slip, and $45^{\circ}$ dip-slip sources are shown after 150 timesteps. The plots have a grey background, where positive is shown in black and negative in white. 
The whole-space finite-difference solutions for the $P-S V$ system are displayed in Figure 5 in the form of divergence and curl. Note that the divergence is nonzero where there is compressional wave energy, and the curl is nonzero for shear wave energy. The far-field radiation patterns are the expected ones. For example, in the strike-slip case, the outgoing $P$ wave has the classic two-lobe pattern while the $S V$ wave has a four-lobed pattern. The complex near-field pattern can be seen in the inner clover leaf followed by a 12-lobed pattern at the source box boundary. Note that this complex pattern changes with time but is required to produce the proper far-field behavior as discussed earlier. The corresponding patterns for the tangential displacement fields for the strike-slip and dip-slip cases are given in Figure 6 . These solutions are considerably simpler. Note that this finite-difference excitation is appropriate for a homogeneous region which was assumed for convenience. However,

\section{SH VERTICAL RADIATION PATTERNS}

$$
t=125 \text { time steps }
$$

$t=250$ time steps
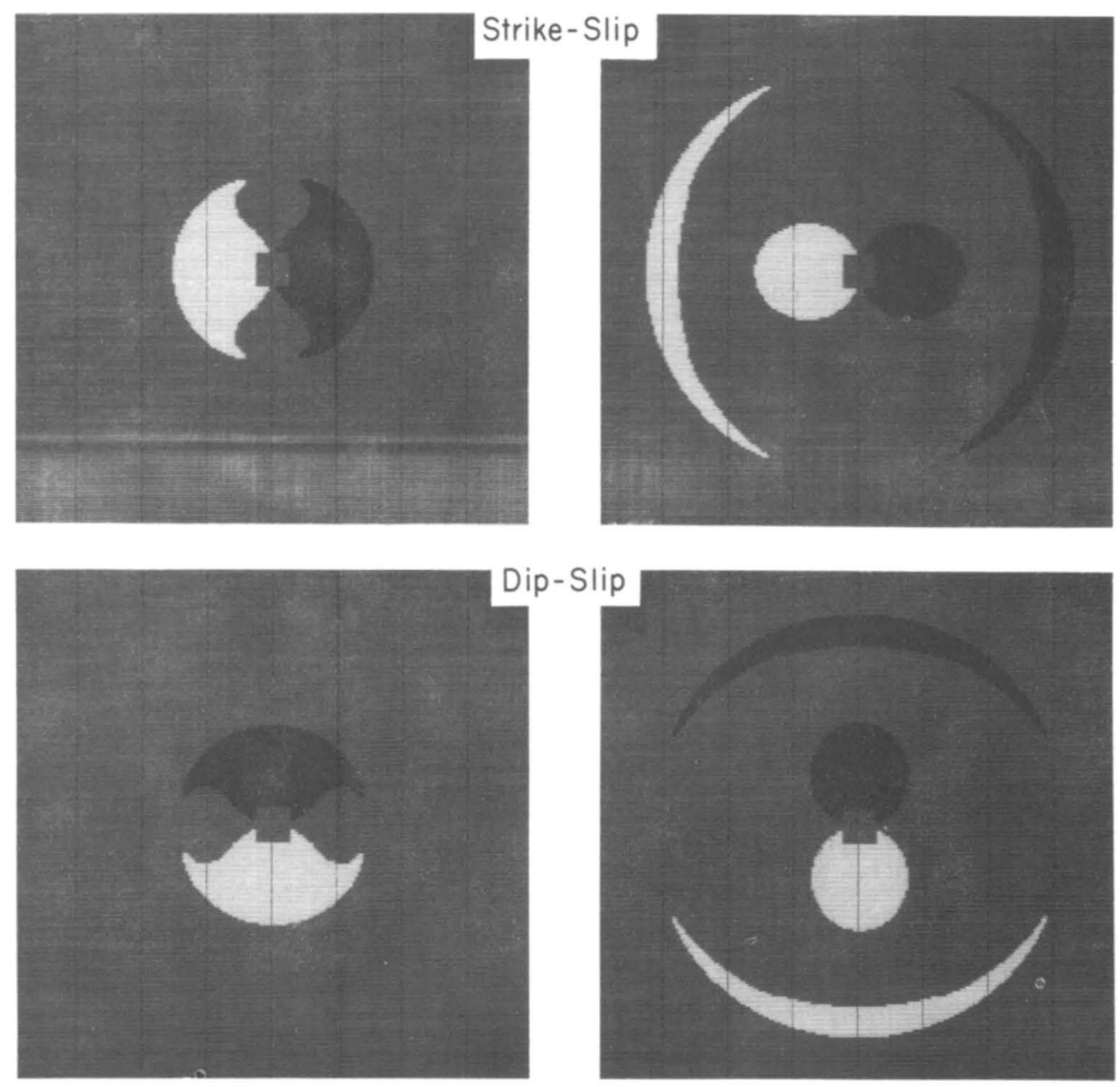

FIG. 6. $S H$ vertical radiation patterns. The displacement field due to strike-slip and dip-slip sources are shown after 125 and 250 timesteps. The plots for the earthquakes have a grey background, where positive displacements are shown in black and negative in white. 
with the help of generalized-ray theory, we could load this source box in a layered structure if desired.

After the energy propagates across the structural model to the receiver (as shown in Figure 1b), we extract the vertical, radial, and transverse motions from the finitedifference code indicated by the symbols $\tilde{W}, \tilde{V}$, and $\tilde{Q}$ for each of the fundamental orientations. Finally, the point-source synthetic seismogram is constructed at the observation point by evaluating

$$
\begin{aligned}
& \boldsymbol{W}_{p}=\frac{1}{\sqrt{R}} \frac{d}{d t}\left[\frac{1}{\sqrt{t}} *\left(A_{1} \tilde{W}^{1}+A_{2} \tilde{W}^{2}+A_{3} \tilde{W}^{3}\right)\right] \\
& \boldsymbol{V}_{p}=\frac{1}{\sqrt{R}} \frac{d}{d t}\left[\frac{1}{\sqrt{t}} *\left(A_{4} \tilde{V}^{1}+A_{5} \tilde{V}^{2}\right)\right] \\
& \boldsymbol{Q}_{p}=\frac{1}{\sqrt{R}} \frac{d}{d t}\left[\frac{1}{\sqrt{t}} *\left(A_{1} \tilde{Q}^{1}+A_{2} \tilde{Q}^{2}+A_{3} \tilde{Q}^{3}\right)\right]
\end{aligned}
$$

where the azimuthal radiation patterns are defined by

$$
\begin{aligned}
& A_{1}=\sin 2 \theta \cos \lambda \sin \delta+\frac{1}{2} \cos 2 \theta \sin \lambda \sin 2 \delta, \\
& A_{2}=\cos \theta \cos \lambda \cos \delta-\sin \theta \sin \lambda \cos 2 \delta, \\
& A_{3}=\frac{1}{2} \sin \lambda \sin 2 \delta, \\
& A_{4}=\cos 2 \theta \cos \lambda \sin \delta-\frac{1}{2} \sin 2 \theta \sin \lambda \sin 2 \delta, \\
& A_{5}=-\sin \theta \cos \lambda \cos \delta-\cos \theta \sin \lambda \cos 2 \delta,
\end{aligned}
$$

where $\theta$ is the strike from the end of the fault plane, $\lambda$ is the rake angle, and $\delta$ is the dip angle (as shown in Figure 2). The same definitions have been used in previous source discussions [see Langston and Helmberger (1975)].

\section{CONCLUSIONS}

A procedure for constructing synthetic seismograms appropriate for earthquake sources using two-dimensional finite-difference codes is discussed. It is based on breaking down the three-dimensional motion field produced by shear dislocations into $S H$ and $P-S V$ systems which can be solved independently applying the twodimensional wave equations. Note that the line sources must be aligned perpendicular to the structure such that no $S H-S V$ mixing is allowed as the motion propagates through the model. Line-source $S H$ and $P-S V$ excitation functions are derived using Cagniard-de Hoop theory for the three principal faults which allows an arbitrarily oriented fault to be simulated by linear summation. Numerical tests of this new technique against analytical codes show good agreement [e.g., see Figure 7 and Vidale and Helmberger (1987)].

The main disadvantage of this approach is the computational expense in running the finite-difference routines and the banded-limited nature of the results. The latter limitations are caused by grid size constraints which control the highest frequency allowed and by the asymptotic approximations at the lowest frequency. The main advantage of this technique over other analytical methods is that it is 


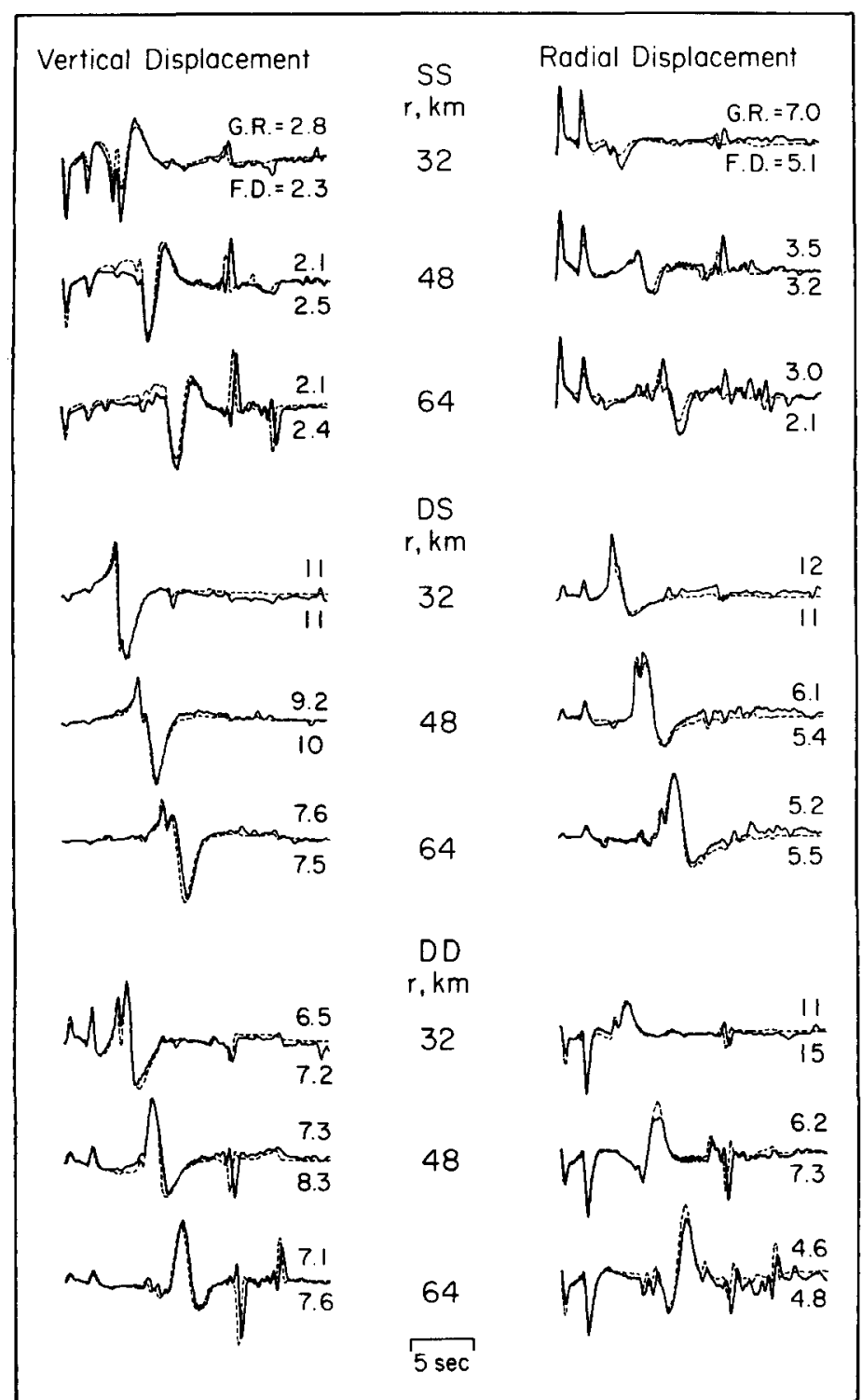

Fig. 7. Comparison of finite-difference (solid) and GRT (dashed) seismograms for the ranges 32,48 , and $64 \mathrm{~km}$ for the strike-slip, dip-slip, and $45^{\circ}$ dip-slip mechanisms, respectively. The far-field source time functions, $\dot{D}(t)$, is specified by a trapezoidal shape with equal $\delta t_{j}$ 's of $0.2 \mathrm{sec}$. The parameter for the layers are given in Table 1 with a source depth of $8 \mathrm{~km}$. Amplitudes may be scaled to moment.

unique in allowing the seismic investigation of complex models such as crosssections through basins and ridges. The usefulness of this scheme is illustrated in Vidale and Helmberger (1988).

\section{ACKNOWLEDGMENTS}

We thank Richard Stead, Heidi Houston, and Gladys Engen for their reviews, and Robert Clayton for his efforts in developing the original finite-difference code. This work was supported by the Geophysical Sciences Division of the Air Force Geophysics Laboratory under Contract F19628-85-K0017 and monitored by the Defense Advanced Research Projects Agency. Contribution no. 4458, Division of Geological and Planetary Sciences, California Institute of Technology. 


\section{REFERENCES}

Archuleta, R. (1984). A faulting model for the 1979 Imperial Valley, California, earthquake, J. Geophys. Res. 89, 4559-4585.

Harkrider, D. (1964). Surface waves in multilayered elastic media. I. Rayleigh and Love waves from buried sources in a multi-layered elastic half-space, Bull. Seism. Soc. Am. 54, 627-679.

Hartzell, S. and T. Heaton (1983). Inversion of strong ground motion and teleseismic waveform data for the fault rupture history of the 1979 Imperial Valley, California, earthquake, Bull. Seism. Soc. Am. $73,1553-1583$.

Helmberger, D. (1968). The crust-mantle transition in the Bering Sea, Bull. Seism. Soc. Am. 58, 179214.

Helmberger, D. (1974). Generalized ray theory for shear dislocations, Bull. Seism. Soc. Am. 64, 45-64.

Helmberger, D. and D. Harkrider (1977). Modeling earthquakes with generalized ray theory: modem problems in elastic wave propagation, IUTAM Symposium, John Wiley \& Sons, New York, 479518.

Langston, C. A. and D. V. Helmberger (1975). A procedure for modeling shallow dislocation sources, Geophys. J. R. Astr. Soc. 42, 117-130.

Olson, A. H. and R. Apsel (1982). Finite faults and inverse theory with applications to the 1979 Imperial Valley earthquake, Bull. Seism. Soc. Am. 72, 1969-2002.

Vidale, J. E. and D. V. Helmberger (1987). Seismic strong motion synthetics, in Computational Techniques, vol. 4, Academic Press, Inc., Orlando, Florida, 267-317.

Vidale, J. E. and D. V. Helmberger (1988). Elastic finite-difference modeling of the 1971 San Fernando, California, earthquake, Bull. Seism. Soc. Am. 78, 122-141.

Vidale, J. E., D. V. Helmberger, and R. W. Clayton (1985). Finite-difference seismograms for $S H$ waves, Bull. Seism. Soc. Am. 75, 1765-1782.

SEISMOLOGICAL LABORATORY, 252-21

California Institute of TeChNology

Pasadena, California 91125

Manuscript received 30 March 1987 